\title{
Reducing suicidal behaviour in people with complex needs: Addressing the challenge of implementing dialectical behaviour therapy
}

\author{
By Michaela A. Swales
}

\begin{abstract}
Dialectical Behaviour Therapy (DBT) is an evidence-based psychological therapy, originally developed for the treatment of people with a diagnosis of borderline personality disorder, that specifically focuses on the treatment of suicidal and self-harm behaviours. This paper describes how DBT, uniquely, both treats long-standing factors contributing to suicidal and self-harm behaviour as well as providing an explicit protocol for treating suicidal crises when they arrive. Despite its success in treating suicidal and self-harm behaviours DBT presents some challenges to implementation in routine practice. The paper concludes by outlining these difficulties and how to solve them.
\end{abstract}

Dialektisk atferdsterapi (DBT) er en evidensbasert psykoterapimetode som opprinnelig ble utviklet som en behandling for mennesker med emosjonelt ustabil personlighetsforstyrrelse med særlig fokus på selvmordsog selvskadingsatferd. Denne artikkelen beskriver hvordan DBT spesifikt behandler både vedvarende risikofaktorer som bidrar til selvmords- og selvskadingsatferd, og akutte selvmordskriser når de oppstår. Til tross for at Dialektisk atferdsterapi har betydelig effekt i behandlingen av selvmords- og selvskadeatferd, byr DBT på noen utfordringer for implementering i daglig klinisk praksis. Artikkelen diskuterer disse utfordringene og hvordan de kan løses.
DIALECTICAL BEHAVIOUR THERAPY (DBT) is a comprehensive, cognitive-behavioural treatment, incorporating mindfulness, that was first used to treat adults with a borderline personality disorder (BPD) diagnosis in the late 8 os and early gos. As the initial RCT was the first to demonstrate success in treating a known to be hard to help group of people (Linehan et al., 1991), DBT was widely disseminated and researched. Extensive evidence now supports the efficacy of DBT as a treatment for clients with BPD and suicidal behaviour (StoffersWinterling et al., 2012; Oud et al., 2018; Miga et al., 2019). As a result, DBT features as a recommended treatment in some jurisdictions (National Institute for Health and Clinical Excellence (2009)) for clients with these problems. In addition, because of the success of the treatment for this client group, DBT has been utilised to treat other presenting problems where emotion dysregulation and suicidal behaviour are central features, for example, adolescents presenting with suicide and self-harming behaviours (Mehlum et al., 2014; McCauley et al., 2018), substance-use disorders (Axelrod, 2019, for review), complex eating disorder presentations (Bhatnagar, Martin-Wagar, \& Wisniewski, 2019). This paper begins by summarising key features 


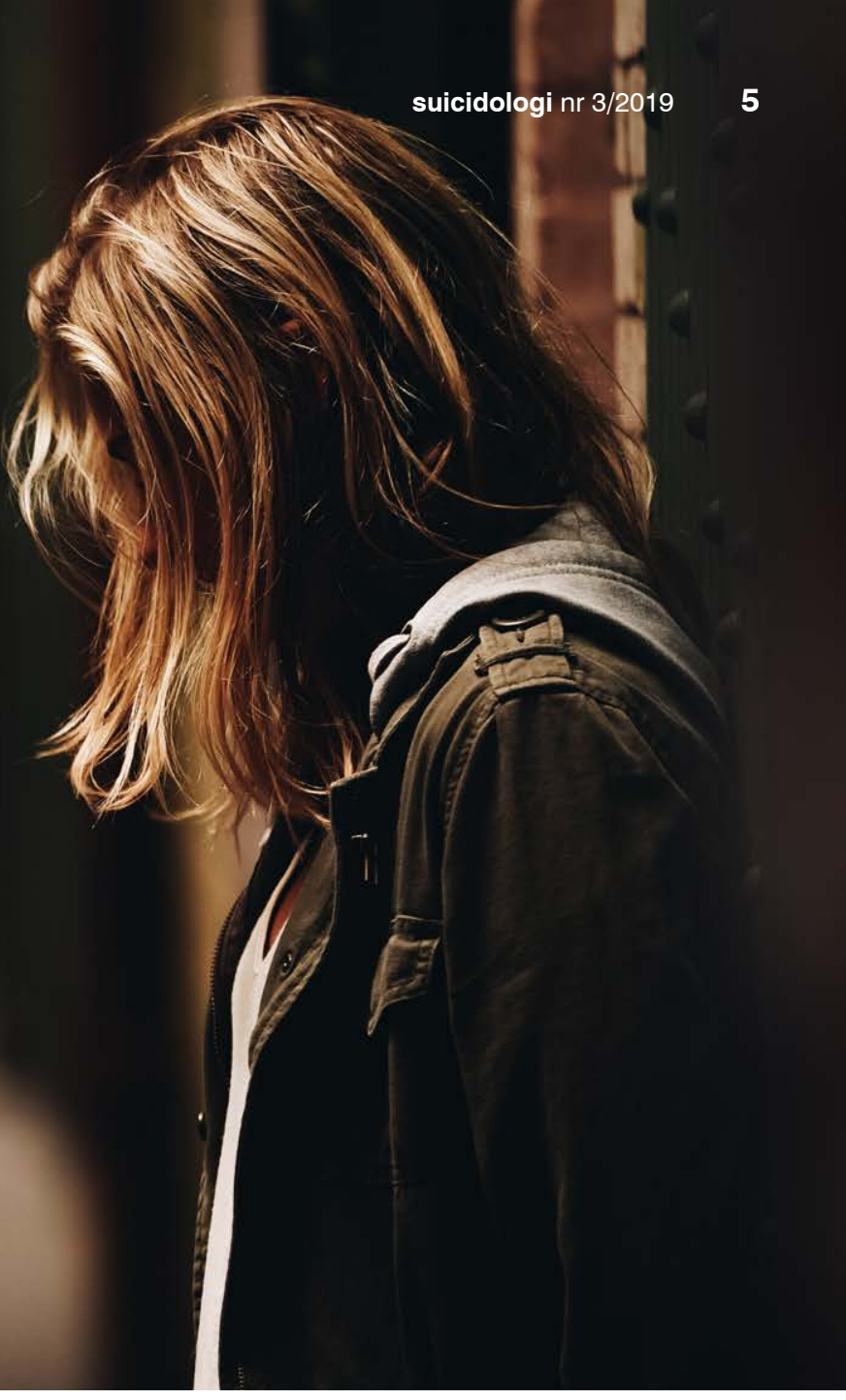

of DBT relating specifically to the treatment of suicidal behaviour. Structural features of the treatment are then described before challenges and solutions to implementing this efficacious treatment into routine clinical practice are considered.

\section{DBT as a treatment for suicidal behaviour}

In the 8os, Marsha Linehan, a suicidologist and a behaviourist, wanted to develop a behavioural intervention to treat suicidal behaviour. Linehan chose to work with clients with a BPD diagnosis precisely because of their high rates of suicidal and self-harm behaviour. So, whilst DBT is often considered as a treatment for BPD, which it is and for which the evidence base is strongest, it began as and still remains, a treatment for chronic suicidal behaviour. As such, DBT has features that may be more broadly applicable to the treatment of recurrent suicidal behaviour, wherever it occurs, and not just in the context of BPD. From the perspective of treating suicidal behaviours DBT has two important features. Uniquely, DBT combines both a specific treatment approach to treating the long-standing issues that lead to and prompt suicidality with a structured protocol linked to the treatment that guides therapists in how to respond in a suicide crisis (Linehan, 1993, chapter 15). Most other treatments focus either exclusively on the former, and utilise treatment as usual in a suicide crisis, or on the latter not attending to longer standing issues. The combination of both of these approaches, with the specific focus on suicidal behaviour, maybe in part what contributes to DBT's efficacy and effectiveness in the treatment of suicidal populations. Indeed, a recent dismantling study concluded that, whilst comprehensive DBT maybe superior at follow-up, less comprehensive forms comprising systematic treatment of suicidal behaviour alongside DBT Skills Training were equally effective in the shorter term (Linehan et al., 2015). DBTs treatment of suicidal behaviours will now be considered in more detail.

\section{Targeting and treatment of suicidal behaviour}

In the first few sessions of treatment, DBT therapists develop a hierarchy of treatment targets to guide the therapeutic focus. Given that people with a BPD diagnosis have one of the highest rates of suicidal behaviour and indeed completed suicide (Gunderson, 2001; Pompili et al., 2005), suicidal behaviours and non-suicidal self-injury (the strongest predictor of subsequent suicide) 


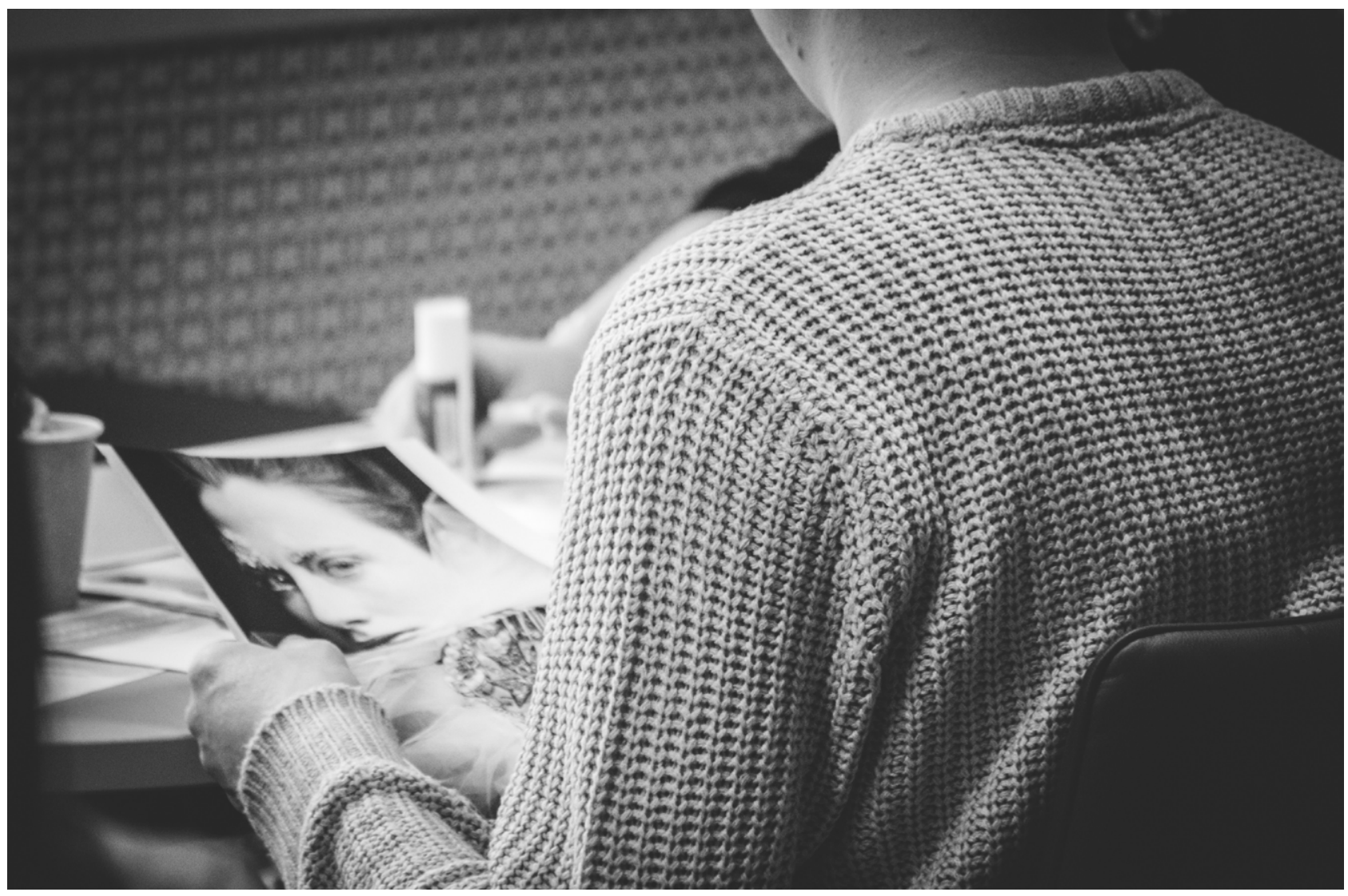

are considered the top targets for therapeutic intervention. These behaviours are followed by therapy-interfering behaviours, e.g. non-attendance or non-compliance with therapy tasks, and quality-of-life interfering behaviours, which are behaviours that are seriously de-stabilising, often warranting intervention from others and behaviours that comprise other psychiatric diagnoses. For example with a client with BPD and co-morbid PTSD, the therapist may target flashbacks and hypervigilance. By addressing these behaviours, DBT also treats other co-morbid conditions that frequently present in clients with a personality disorder diagnosis.

Clients in DBT monitor their agreed target behaviours each week enabling the therapist to select the highest priority behaviour for analysis and treatment. DBT therapists conduct behavioural and solution analyses of this behaviour, investigating prompting events and the emotions, thoughts and actions leading up to and following the target behaviour. Broadly speaking, DBT conceptualises problem behaviours as solutions to emotional problems. Thus, DBT therapists aim to identify, across target behaviours, common patterns of problematic emotions that both drive the occurrence of behaviours on the antecedent side and maintain behaviours on the consequent side. As behaviourists, they are alert to the presence of both classical and operant conditioned responses in clients' repertoires of behaviours.

Utilising four broad classes of 'change' procedures DBT therapists collaboratively develop solution analyses for these controlling variables that contribute to the occurrence and maintenance of the suicidal behaviour. DBT change procedures encompass: a comprehensive set of skills, taught in a weekly skills class, to manage crises, regulate emotions and improve interpersonal effectiveness (Linehan, 2015); cognitive restructuring techniques; exposure and contingency management procedures. In selecting the most appropriate solutions for controlling variables, DBT therapists assess for capability and motivational deficits. In many cases clients lack basic skills in managing emotions or asking for help. In these cases, DBT therapists teach and shape a new repertoire of skills based on those taught in the weekly skills class. Almost all clients, in addition to lacking basic capacities, have significant motivational difficulties such that, even if the skill is in their repertoire, they do not use it when it would be appropriate to do so. Frequently, problematic cognitions, unwarranted emotions and/or punishing environmental contingencies interfere with skilful behaviour. In these circumstances, based on their analyses, DBT therapists deploy cognitive restructuring, exposure and contingency 
Cara is 25 years old. She was referred to her local DBT programme after several psychological treatments (cognitive-behaviour therapy, interpersonal therapy) failed to improve her difficulties. She has a diagnosis of borderline personality disorder, eating disorder not otherwise specified, and a history of trauma. She has been in DBT for 8 weeks. Her primary targets to decrease in therapy are: overdosing on paracetamol and prescribed medication, cutting her arms and thighs, bingeing, vomiting, flashbacks. Targets to increase are: attendance at therapy appointments, implementing solutions generated in therapy, attendance at college.

This week in therapy Cara reports an overdose of tablets. During a night out with friends, Cara met a man who seemed interested in her. Cara became increasingly anxious as they talked more, and she began to worry that he might rape her. She left the club abruptly. She felt ashamed and besieged by self-critical thoughts. She texted her sister and described what happened. Her sister told her not 'to be so stupid' and that the guy was 'just wanting to get to know her better'. Cara's shame and self-criticism intensified. She began ruminating on several failed relationships, and became increasingly hopeless and suicidal. She took 30 paracetamol, hoping that this would kill her, and went to bed. Shortly afterwards, her friend from the nightclub arrived back at the flat, discovered the pill packets and called an ambulance. Cara was admitted to hospital and received treatment. Her sister came to the hospital the following day and apologised for not understanding her.

In analysing this sequence of events, the therapist and Cara identified that the controlling variables for the overdose were the intense shame and self-criticism; hopeless thoughts about the future; and family members only validating her after she has harmed herself. In this session, the therapist, in discussion with Cara, elected to work on the shame and accompanying self-criticism as targets, as these had previously featured as controlling variables in relation to other targeted behaviours (bingeing and vomiting). Together they identified that the majority of Cara's shame was unwarranted; mild embarrassment about rushing away from her friends was all that was warranted. Emotion regulation skills for unwarranted shame were taught and rehearsed in session, including rehearsing alternative more mindful cognitions as an alternative to self-critical thoughts. At the end of the session, they rehearsed skills for regulating anxiety sufficiently to access support from her friends, in the future, to assess if a situation was safe. They decided to leave to another occasion assessing whether Cara's family's interactional style could be changed. Before the session ended the therapist established that Cara no longer had a hoard of tablets in her flat. Thankfully the friend had removed all the other tablets Cara had collected. management procedures respectively. An example of a behavioural and solution analysis is provided in Text Box 1.

To complement this relentless focus on behaviour change, DBT therapists also utilise several strategies likely to assist in the mitigation of suicide risk. Therapists deploy validation strategies as a counterpoint to pushing for change. In locating and validating those aspects of clients' responses that make sense in their current context, DBT therapists forge effective working alliances that can reduce the isolation that suicidal clients often experience. DBT therapists also have a reciprocal therapeutic style, emphasising warmth, genuineness and self-disclosure that aims to further enhance the therapeutic connection which is of essential importance with suicidal clients (Mehlum, 2019).

\section{Treatment in a suicide crisis}

In addition to the weekly focus on solving problems that lead to suicidal and non-suicidal self-injury behaviours, DBT has a structured protocol to utilise when a client presents in crisis either in-session or on the telephone (Linehan, 1993, chapter 15). Whilst this protocol has been part of DBT since its inception, only recently has it become known more widely outside of DBT as the Linehan Risk Assessment and Management Protocol (LRAMP) and in its web-based version as the Linehan Suicide Safety Net (Harned et al., 2017).

DBT strongly emphasises maintaining clients in the community and does not advocate hospitalisation in a suicidal crisis except in exceptional circumstances. In order to facilitate maintaining community treatment and ensuring clients' safety, DBT therapists using the protocol, in whatever format, follow a series of steps to analyse and treat the crisis. First, the therapist assesses for imminent risk of action to end life. Clients in DBT are by default in a high-risk group and so the therapist is not attending here to well-known demographic risk factors, as these are already known. Rather they systematically focus on imminent risk factors known at a population level, for example, current suicide planning, access to means, attempts to prevent discovery, supplemented with ideographic risk factors, which they know were causal factors in previous suicidal behaviours of the client. Second, the therapist focuses on solving the problem that prompted the current suicide response. This is not a moment to address long-standing issues but to attend to the current crisis. Often this may be an 
interpersonal conflict or some other distressing external event that has overwhelmed the client's coping skills. Alternatively, it may be an extremely intense level of emotion that the client either has no skills to solve or their energy to continue managing their emotional state has faded. In these discussions the therapist continually focuses on solving the problem that resulted in the client actively planning suicide. DBT conceptualises suicidal behaviour as a form of problem-solving; in the context of extreme emotional distress, with limited other solutions to their problems available, suicide is the only strategy from the client's perspective that can terminate the current crisis. Recognising that suicide is essentially an escape plan - the mission of the therapist is to create and develop, as collaboratively as possible with the client, a non-suicidal set of alternative, workable solutions to the client's crisis. Third, DBT therapists attend to high-risk behavioural indicators. They will directly address the removal of lethal means - and may even postpone their problem-solving efforts until this has been accomplished. DBT therapists will also directly challenge any inaccurate perceptions about suicide and confront fantasies about death by suicide in order to decrease motivation for suicide as a solution. Fourth, and in counterbalance to the strong problem-solving focus, therapists will help the client to develop affect tolerance, primarily by validating the emotional pain of the client, the natural desire to want to escape such pain and by instructing in DBT skills that will aid the tolerance and regulation of emotional distress. Therapists then move to gain a commitment to the non-suicidal plan of action developed in the discussion before concluding with a re-assessment of the imminence of the suicide risk and finally, trouble-shooting what to do if the suicidal urges recur. An example of a typical crisis call is given in Text Box II.

\section{Structural features of DBT}

Before considering implementation of DBT in routine practice, it is worth considering structural aspects of the treatment relevant to its implementation. DBT has a programmatic structure and, as a consequence of its multi-modal structure, teams of professionals deliver DBT. In traditional out-patient settings, DBT comprises

\section{TEXT BOX II}

Cara texts her therapist at 8pm on a mid-week evening, saying 'skills not working, it's all pointless'. On receiving the text, Cara's therapist returns the call. She opens by asking directly about Cara's suicidal urges, knowing from past experience that the word 'pointless' is often associated with suicidal actions. Cara confirms that her urges are high and that she has access to medication that she intends to take. Her therapist, first, negotiates disposal of the means. Cara is reluctant to give up the medication. Her therapist reminds her that planning to overdose is Cara's way of trying to solve a problem and that the therapist will help her solve whatever the problem is but can only do so when she is assured of Cara's safety. Reluctantly Cara flushes the medication down the toilet - which the therapist listens on the phone. Then the therapist asks Cara to describe briefly the events leading to the suicidal crisis. Cara describes discovering that some members of her family are planning a weekend away and have not invited her. Cara reports that this is because she is such a dreadful sister and daughter. The therapist irreverently comments that if she wishes to not be a dreadful daughter and sister then she should definitely not commit suicide! In response, Cara becomes more engaged in active problem-solving, opening up the opportunity for the therapist to drop her matter-of-fact voice tone and become more validating and warmer as Cara participates more in skilful behaviour. On further enquiry by the therapist, Cara acknowledges experiencing intense sadness (about not being invited) and shame (because she believes it is her fault). The therapist works on helping Cara apply opposite action skills for shame (as these have previously been rehearsed in session) whilst then guiding her through the check the facts skill in relation to the reason for her not being invited. The therapist reassures Cara that if it is the case, as Cara fears, that she has not been invited because of her past behaviours, the therapist will provide further coaching on how to handle these distressing emotions and how to change her behaviours. At the end of the call Cara reports that her suicidal urges have gone and she feels more hopeful that she can cope with the situation. The therapist asks Cara to rehearse what she will do if the urges return. They schedule a check-in call the following day so the therapist can hear about the progress Cara has made in checking the facts with her family. During the check-in call, Cara reports that her sister confirms that they had not invited her because she had refused a similar offer the previous year, saying she was too anxious to go away and she would be a 'nuisance' to everybody. Her sister has asked her if she would like to go. Cara is now highly anxious about whether to go and how she will cope. The therapist asks her to try and find her Wise Mind about whether to go or not, and that they can work on this more in their scheduled session, including plans for going away using the cope ahead skill, early the next week. 
four treatment modalities, three of which are client facing (Linehan, 1993). Clients participate in weekly skills groups that typically last for 2.5 hours during which two skills trainers teach and strengthen new skills in mindfulness, interpersonal effectiveness, emotion regulation and distress tolerance (Linehan, 2015). Individual DBT therapy sessions are also held weekly. In these session, DBT therapists conduct the thorough behavioural and solution analysis of factors contributing to suicidal and self-harm behaviours (Rizvi, 2019). Clients also have access to their individual therapist outside traditional office hours, and within the therapists' limits, for telephone coaching in how to apply the newly learned skills in the everyday challenges of their

lives. Whilst contact between sessions is

These modalities of DBT essentially fulfil four key functions. most commonly by phone, in recent years texts and emails are increasingly utilised as methods to aid generalisation of skills

(Chapman, 2019). Access by phone between sessions is yet a further mechanism by which DBT endeavours to treat suicidal behaviours. By providing assistance as and when needed, therapists can intervene earlier in a sequence of problems that might otherwise lead to a suicidal behaviour. Clients are instructed in how to effectively utilise the phone early in treatment and oriented to the fact that they will not receive therapy on the phone, rather they can access coaching in using skills. If clients do take suicidal action, access to the therapist by phone is withdrawn for 24 hours. Data has shown that offering such access, compared to therapists offering other treatments, does not increase the number of calls therapists receive; it removes the correlation between phone calls and suicidal communication, and, typically, calls last about 6 minutes (Chapman, 2019; Rizvi \& Roman, 2019). In offering out-of-hours access, DBT therapists also signal availability to provide help and support when the client is in difficulty. This feature of the treatment, recognised as a requirement in the field of suicide prevention (Mann et al., 2005) may also contribute to decreasing isolation and risk in the face of a suicidal crisis.

The final modality of treatment, DBT Consultation Team meeting, provides an opportunity for therapists to meet weekly to receive assistance in delivering the treatment (Sayrs \& Linehan, 2019). Typically, the consultation team meeting lasts for two hours a week. During one of these hours, therapists request consultation on how they may improve the quality of the treatment that they offer. The focus during the consultation is on how the team can shape therapists' behaviour to be maximally effective with the clients rather than on discussing client progress, as is typical in a standard case discussion meeting. The style of the meeting requires a willingness from DBT therapists to expose their work with clients to feedback from colleagues and be open to changing their own behaviour in the service of improved client outcomes. The second hour focuses on teaching and developing further skills in the therapy.

This four-mode structure works well in typical out-patient settings. These modalities of DBT essentially fulfil four key functions: enhancing client capabilities (skills training groups), enhancing client motivation (individual DBT psychological therapy), ensuring generalisation of treatment gains (telephone coaching), and enhancing therapists' capabilities and motivation (consultation team meeting). A further fifth function, structuring the environment, becomes relevant with other client groups and in other contexts (Linehan, Cochran, \& Kehrer, 2001). For example, adolescent programmes, as well as including parents in the skills classes, frequently involve parents in family sessions examining wider systemic factors that impact the clients' target behaviours (Miller, Rathus, \& Linehan, 2007). In inpatient settings, modalities addressing the reinforcing and punishing consequences of the milieu may be highly relevant to the success of the programme (Fox, 2019). In these circumstances, the DBT team attends to ensuring that the wider environment, either of the clinical service or the client's home environment reinforces treatment gains.

\section{Implementing DBT: Challenges and Solutions}

In addition to evidence of efficacy, DBT also effectively delivers improved clinical outcomes in routine practice (Walton \& Comtois, 2019). Despite evidence of effective translation of the treatment from the 'laboratory' to routine practice, implementing DBT in healthcare services presents a number of challenges. It requires mental health professionals to be configured in teams that can meaningfully work together to deliver a programme of treatment, capacity to deliver an intensive treatment (multiple modalities across the week) for extended treatment periods (up to a year), and strategies to sustain the programme. In recent years, given the extent of the dissemination and implementation effort in DBT, clinicians and researchers have discussed in more detail the implementation challenges and how they may be addressed (Comtois \& Landes, 2019; Toms et al., 2019).

\section{Making a case for DBT: Organisational Pre-treatment} Given the extent of organisational change required to install a DBT programme, preparing the organisation, much as you would a client, for treatment is advisable. Similar steps to this process have been identified (Swales, 2010). First, as 'goal fit and suitability' has been identified as a potential implementation barrier for DBT (Toms et al., 2019), whoever is leading the proposed implementation must establish the goals or stated aims of the organisation proposing to host the programme. DBT fits well in organisations that routinely provide therapeutic interventions, that frequently see suicidal 


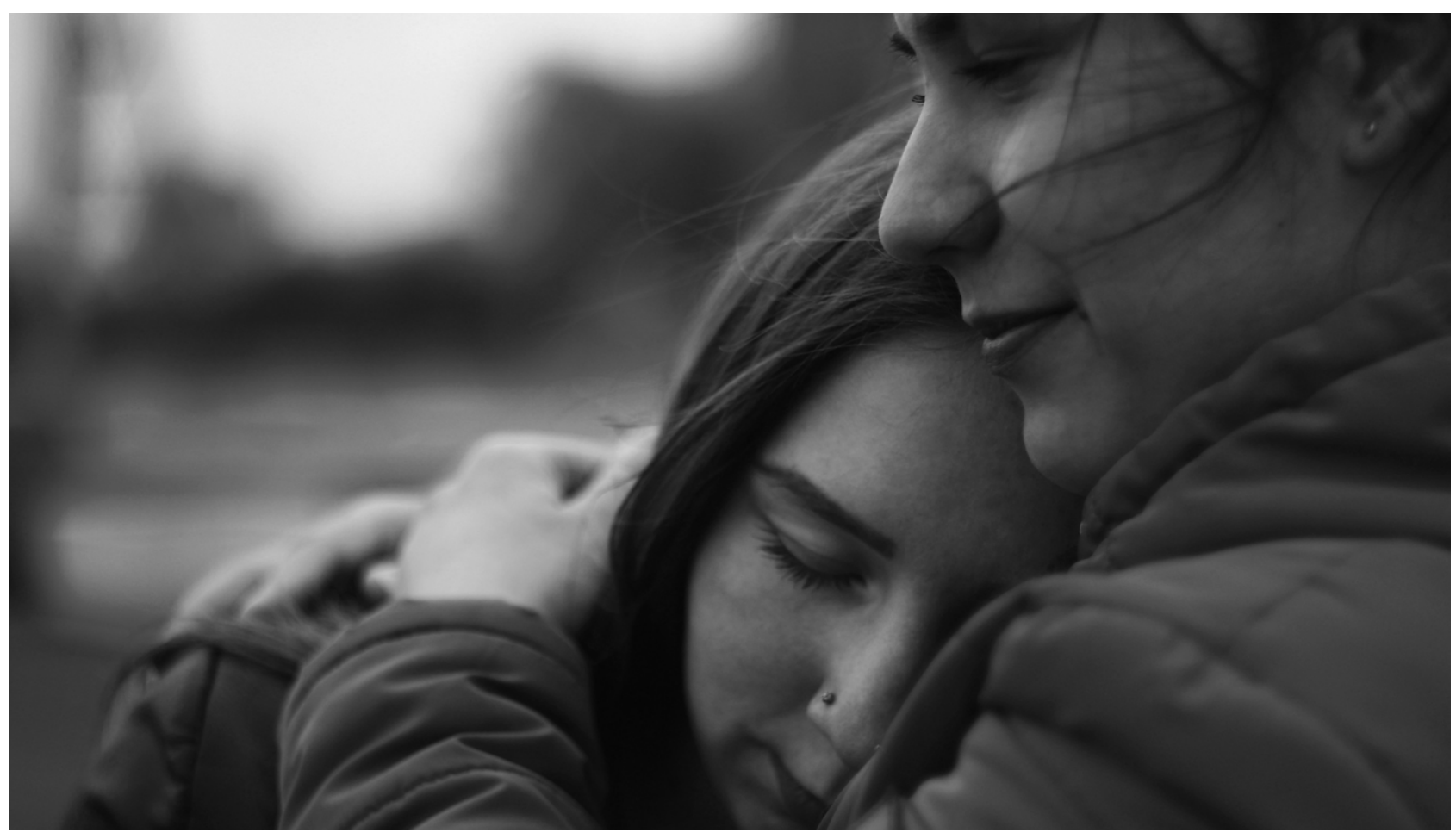

clients and have staff trained as mental health professionals. Organisations seeking directly to reduce suicidal behaviours or whose societal role could be considered to deliver on government targets, for example public sector mental health services, are ideally suited to deliver DBT programmes. Explicitly linking DBT's goals of reducing suicidal behaviours and improving quality of life to the goals of the organisation is a necessary first step. Implementers must beware, however, of assuming that a good match between the organisation's goals and those of DBT is sufficient for effective implementation. Organisations have multiple goals, some of which may conflict with those of DBT. For example, whilst in the UK there are clear directives for NHS services to reduce suicide and suicidal behaviours, there are also equally strong directives to reduce waiting times, making delivery of a time and personnel intense treatment a low priority for some parts of the system. Whilst DBT may decrease service utilisation (Krawitz \& Miga, 2019), the places where it decreases demand e.g. unscheduled care services, inpatient services, may not be those where the savings can easily be reinvested in outpatient mental health services. So, ensuring that the organisation has signed up for DBT requires that any conflicting goals are identified and addressed and management within the system can agree that adopting DBT is an appropriate treatment option to implement.

Before implementing DBT, practitioners may find it salutatory to review their organisations previous history of implementing new services or treatments. Some

\section{A further fifth function, structuring the environment, becomes relevant with other client groups and in other contexts.}

organisations may excel in successful implementation and in such cases DBT implementers would benefit from discussions with leaders of previous implementations about factors within the system that ensured their success. Indeed, organisations with higher ratings of organisational cohesion and communication are more likely to implement more modalities of DBT (Ditty et al., 2015). Where the organisation has a history of failed implementation, DBT implementers are advised to interview several people associated with previous attempts at innovation and change to identify barriers encountered and to make a strategic plan about how to address them. In all cases forming a steering group of all relevant stakeholders, to build collaboration (Koons et al., 2006) and increase buy-in to the intervention will increase the likelihood of successful implementation and is a recognised implementation strategy (Powell et al., 2012).

\section{Programme Installation}

Once organisational agreement has been finalised there are several steps involved in installing the programme and beginning to deliver a DBT service. 
Training - who to train, team leadership. Several studies indicate that staff from a variety of backgrounds can learn and deliver DBT (Hawkins \& Sinha, 1998). Given the treatment's behavioural focus, teams require that at least some practitioners have knowledge of the application of behavioural principles before they commence training especially as this has been highlighted as an implementation challenge (Hawkins \& Sinha, 1998). In addition, some studies of DBT indicate that selecting staff who are non-judgemental with more positive attitudes towards BPD correlates with increased use of DBT (Herschell et al., 2014). Selecting staff who embody a stance of equality combined with skills in group management and teaching has also been described as helpful (Cunningham, Wolbert, \& Lillie, 2004).

Training facilitates implementation and increased training is associated with improved confidence and greater use of DBT (Frederick \& Comtois, 2006; Herschell et al., 2014; Swales, Taylor, \& Hibbs, 2012). Intensive Training ${ }^{\mathrm{TM}}$ is the most researched training model (DuBose et al., 2019). In this form of training the team planning to deliver the treatment train together, attending two five-day seminars separated by 6-8 months. During the first five days of training, practitioners receive didactic instruction, augmented by experiential practices, to learn the key principles and strategies of the treatment. Before the second part of the training, teams must implement the treatment - setting up a DBT programme - returning with presentations about their new service and the challenges encountered in set-up and a clinical case that they have treated within their service. Practitioners receive coaching and feedback to shape further skill and implementation of the treatment. This unique training model, focusing on teams and explicitly requiring implementation, counteracts the 'train and hope' strategy common in many public sector implementations where organisations only focus on training staff and 'hope' that they will implement what they have learnt (Fixsen et al., 2005). Requiring teams to implement before returning to the second part of training may account for DBT's success in dissemination and implementation (McHugh \& Barlow, 2010). On-going consultation post-training has also been found to facilitate DBT implementation (Carmel, Rose, \& Fruzzetti, 2014).

DBT programmes require a team lead (Swales \& Dunkley, 2019). The role comprises two main components; first, to ensure the team delivers an adherent DBT programme and second, to link with the wider organisation to secure resources for the team and ensure accountability of the programme to the organisation. DBT team leads often acquire the position by accident - they were simply the most willing person to organise the training of the team. During training, teams and team leads should consider who has the necessary skills for the role. Team leads will need to have sufficient skill and organisational connections to effectively liaise with the wider organisation to ensure the team has necessary resources, including funding (Kinsey \& Reed, 2015) and to protect the team as far as possible from wider organisational pressures. Ideally, team leads have a more senior position in the wider system combined with influencing skills. Without this they may struggle to ensure that the team has adequate resources. When a team lead lacks seniority, recruiting a DBT champion from the wider system who has more influence and power to maintain programme resource may prove helpful (Wolpow, Porter, \& Hermanos, 2000). Team leads function best when they also have a high level of skill in the treatment, for example, the capacity to model DBT skills (Little, Butler, \& Fowler, 2010). Their task is to lead the team to deliver an adherent programme and this is a task best done when leading from the front! As a priority, post initial training, team leads should seek supervision and advanced training in DBT to further develop their skills to enable them to more effectively lead the team.

Allocating Clinician Time to the Programme. Prior to training, team members are required to commit to devoting 1.5 days per week to the delivery of the new programme during the training period. Successful installation of a DBT programme requires a genuine commitment and is not simply a paper exercise. Research into implementation of DBT indicates that this is the first point at which installation of a DBT programme can go awry (Swales, Taylor, \& Hibbs, 2012). Whilst DBT programmes survive well during their first year, supported by the structure of the training, some programmes 'die' in the second year. Clinicians commonly cite insufficient time devoted to DBT and competing duties as implementation barriers (Carmel, Rose, \& Fruzzetti, 2014; Herschell et al., 2009; Perseius et al., 2007). Often this situation arises when the goals of senior and middle managers are in conflict. Senior management may support DBT because it can deliver on the goals of the organisation, for example, providing evidence-based care, whereas middle managers, directly supervising the staff, are more directly concerned with other aspects of service delivery, such as waiting times, and refuse to agree to the release of staff time to deliver DBT. In these circumstances, practitioners become overwhelmed with the task of implementing DBT, in addition to existing commitments, and often the DBT programme comes to a premature end. Guarding against this problem requires a DBT team lead or the implementation lead, in discussion with the stakeholder group, to give active consideration to what the system needs to de-implement (Wang et al., 2018) in order to free staff time to deliver a new programme. DBT often successfully reduces unscheduled care to the wider mental health team and so does ultimately lead to a freeing up of practitioner time. However, these changes do not come at the start of the programme and if practitioners 
have insufficient time to really learn the new approach the benefits to the clients and the system will never be realised.

Programme Delivery: Implement with fidelity. Teams in training are encouraged to implement programmes as close to the published evidence base as possible. Doing so, increases the likelihood of delivering outcomes comparable to the published RCTs. Sadly, often teams and services focus more on what they think they can deliver rather than on implementing with fidelity. This is a common problem in implementation of any healthcare intervention, and may result in poorer clinical outcomes, yet systems persist in modifying and adapting interventions in the absence of evidence that this is effective (Breitenstein et al., 2010).

Primarily, systems and practitioners seek to adapt rather than adopt with fidelity because of limitations and constraints within their systems. This is understandable, especially in countries and/or systems with low levels of resources. Indeed, even in relatively well funded healthcare systems, there remain financial pressures resulting in questions about how much

\section{Research into DBT implementation indicates that a major challenge remains - the scalability of DBT programmes.}

of a complex treatment to implement. More recent research that supports less complex versions of DBT will likely help in these discussions and debates. For example, there is now evidence of the effectiveness of DBT skills groups alone, especially for clients with less severe presentations (Valentine et al., 2015). Current studies are also examining whether the requisite treatment length needs to be 12 months or whether a shorter duration might also be effective (McMain et al., 2019). Adaptations of DBT in low- and middle-income countries are also now been developed (Ramaiya et al., 2017).

Given that it is not yet known what adaptations of DBT retain the efficacy of the original version, implementation science would advise teams, notwithstanding the challenges, to remain as close to the current evidence base as possible. DBT has been evaluated in a range of settings, with a number of different populations and curricula for these different programmes have been published (Linehan, 2015) to support teams in making an appropriate selection for their setting. Likewise, practitioners are encouraged to teach directly from the manual in the skills group portion of the treatment. Astonishingly, despite the detail, precision, and practical examples within the manual, teams often start trying to amend handouts and change the curricula. Such an approach increases workload and will not necessarily lead to improved client outcomes - in fact the reverse is more likely true. Developing the capacity and willingness to teach the content of the manual as is, requires a particular skill set from practitioners when they implement an evidence-based treatment. If there are pressing reasons for adapting before delivering with fidelity, for example significant cultural differences, teams and practitioners are advised to monitor outcomes to ensure that the effectiveness of the treatment has not been compromised (Kinsey \& Reed, 2015).

In addition to implementing the content with fidelity, implementing the structure with fidelity is advised. Studies indicate that teams most often implement skills training groups, individual therapy and consultation team. Telephone consultation is the least frequently implemented modality (Navarro-Haro et al., 2019). Offering out-of-hours phone calls often clashes with established working practices and, in particular, is at odds with views from other therapy models that with clients with personality disorder being boundaried is vital. Successful implementation of the telephone modality, therefore, requires several steps. First, the system requires orienting to the purpose and function of telephone consultation and that calls are not for therapy but for skills coaching. Second, terms and conditions in staff contracts may need reviewing to ensure that practitioners are not working outside their contract and so remain insured and indemnified by their employers. Third, practitioner hours or pay will require adjustment to compensate for availability out-of-hours. In practice, as calls are so few and short, some allowance for practitioners to flexibly adjust their hours of work if they have been involved in out-of-hours calls probably makes the most sense. Finally, practitioners require a mobile device that they can use for therapy calls, that can also store relevant contact information for clients. Ideally, this is not their personal device. Systems will also need to agree how such out-of-hours contact is recorded in the clinical record and the time frames in which this should be done.

\section{Plan for sustainability and scalability}

DBT Team leads and stakeholder groups need to plan for sustainability of their programme. Turnover in most mental health systems means that any programme needs to consider how to maintain a core group of staff (Carmel, Rose, \& Fruzzetti, 2014; Herschell et al., 2009). Studies of DBT sustainability, indicate that whilst DBT programmes sustain well compared to other evidence-based practices, during the first five years post-training survivability decreases prompted by staff turnover. (King et al., 2018; Swales, Taylor, \& Hibbs, 2012). As DBT is only in exceptionally rare cases taught on professional training programmes, teams need to be prepared to train new staff. When recruiting new staff, knowledge of DBT or a willingness to learn the treatment and deliver it with fidelity must comprise part 
of the job descriptions for posts. New staff hoping to join a programme should go through a 'pre-treatment' intervention to assess for their commitment to learn and deliver the programme. Team leads ideally will coordinate this process.

Research into DBT implementation indicates that a major challenge remains - the scalability of DBT programmes. Whilst DBT has been implemented widely with an estimated 6000 provider teams in 19 countries (DuBose, Botanov, \& Ivanoff, 2019), often DBT programmes are small. Research in the UK indicated that the average number of clients treated by programmes, except in a small number of cases, was less than 10 clients per year (Swales, Taylor, \& Hibbs, 2012). Primarily this problem arises because systems do not think beyond training and only focus on having DBT to offer. Evidence-based commissioning of a DBT service would rest on an analysis of the number of clients for whom DBT would be an effective intervention in a given locality and then scaling the DBT programme to provide that number of places. Rarely do systems act in this way. Persuading service commissioners to actively assess the demand for DBT and build their service response around that, will be an important step in the future development of services. Also, given the intensive nature of the treatment and the extent of suicidal behaviour in the population, provision of comprehensive DBT services to all who potentially could benefit is likely to be unrealistic. Recent research, as discussed earlier, examining the impact of less comprehensive forms of DBT is showing promise for less complex presentations. Future services may comprise a comprehensive treatment programme for the most severe and complex clients presenting to a service, with a lower intensity offering for a broader range of clients.

\section{Conclusion}

DBT is a strongly evidence-based treatment for suicidal behaviour. It began as a treatment solely for clients with BPD and suicidal behaviour, and whilst the strongest evidence-based for DBT remains for this group, other client populations have more recently demonstrated clinical benefits. DBT's focus on suicidal and non-suicidal self-injury behaviours means that it uniquely treats these behaviours rather than simply assessing and managing them. Consequently, DBT should be considered for implementation in services treating clients with complex mental health needs and high-risk suicidal behaviours. Over the last twenty years, several factors have become apparent as relevant in successfully implementing DBT in routine practice. These are: ensuring DBT matches the organisations goals; resolving conflicting organisational goals; train staff in the context of adjusted job plans where they have time to learn and acquire the skills of the treatment; plan for turnover; assess the size of the clinical problem locally and scale the DBT programme accordingly.

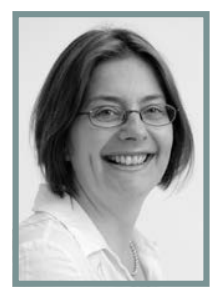

MICHAELA SWALES PhD is a Consultant Clinical Psychologist and Professor in Clinical Psychology on the North Wales Clinical Psychology Programme, Bangor University. Prof. Swales is the Director of the British Isles Training Team, an International Affiliate of the Linehan Institute. She has trained more than a thousand professionals in DBT, seeding over 400 programmes, in both the UK and further afield.

Professor Swales declares the following interests: she receives royalties from books on DBT, income from fees for training and her husband is managing director and major shareholder in a company providing DBT training.

\section{REFERENCES}

Axelrod, S. R. (2019). Dialectical behaviour Therapy for Substance-Use Disorders. In Swales, M. (Ed). Oxford Handbook of DBT. Oxford: OUP.

Bhatnagar, K. A. C., Martin-Wagar, C. \& Wisniewski, L. (2019). DBT for Eating Disorders: An overview. In Swales, M. (Ed). Oxford Handbook of DBT. Oxford: OUP.

Breitenstein, S. M., Gross, D., Garvey, C., Hill, C., Fogg, L. \& Resnick, B. (2010). Implementation Fidelity in Community-Based Interventions. Research in Nursing \& Health, 33(2), 164-173.

Carmel, A., Rose, M., \& Fruzzetti, A.E. (2014). Barriers and solutions to implementing dialectical behavior therapy in a public behavioral health system. Administration and Policy in Mental Health, 41(5), 608 - 614.

Chapman, A. L. (2019). Phone coaching in Dialectical Behaviour Therapy. Guilford DBT ${ }^{\circledR}$ Practice Series. New York: Guilford.

Comtois, K. A. \& Landes, S. I. (2019). Implementing DBT: An implementation science perspective. In Swales, M. (Ed). Oxford Handbook of DBT. Oxford: OUP

Cunningham, K., Wolbert, R., \& Lillie, B. (2004). It's about me solving my problems: clients' assessments of dialectical behavior therapy. Cognitive and Behavioural Practice., 11(2), 248-256.

Ditty, M. S., Landes, S. I., Doyle, A., \& Beidas, R. S. (2015). It takes a village: a mixed method analysis of inner setting variables and dialectical behaviour therapy implementation. Adm Policy Ment Health.42 (6) 672-681.

DuBose, A. P., Botanov, Y., \& Ivanoff, A. (2019). International Implementation of Dialectical Behaviour Therapy: The Challenge of Training Therapists across Cultures. In Swales, M. (Ed). Oxford Handbook of DBT. Oxford: OUP

DuBose, A. P., Botanov, Y., Navarro-Haro, M. V., \& Linehan, M. M. (2019). Evidence-based training: The Intensive model of training in Dialectical Behaviour Therapy. In Swales, M. (Ed). Oxford Handbook of DBT. Oxford: OUP.

Fixsen, D. L., Naoom, S. F., Blase, K. A., Friedman, R. M., \& Wallace, F. (2005). Implementation research: a synthesis of the literature. Tampa FL, America: University of South Florida, Louis de la Parte Florida Mental Health Institute, The National Implementation Research Network (FMHI Publication \#231).

Fox, E. (2019). Delivering DBT in an inpatient setting. In Swales, M. (Ed). Oxford Handbook of DBT. Oxford: OUP.

Frederick, I. T., \& Comtois, K. A. (2006). Practice of dialectical behaviour therapy after psychiatry residency. Academic Psychiatry. 30(1), 63-68.

Gunderson, I. G. (2001). Borderline personality disorder: A clinical guide. Washington, DC: American Psychiatric Association.

Harned, M. S., Lungu, A., Wilks, C. R., \& Linehan, M. M. (2017). Evaluating a multi-media tool for suicide risk assessment and management: The Linehan Suicide Safety Net. Journal of Clinical Psychology, 73(3), 308-318. 
Hawkins, K. A. \& Sinha, R. (1998). Can line clinicians master the concep tual complexities of dialectical behavior therapy? An evaluation of a state department of mental health training program. Journal of Psychiatry Research, 32(6), 379-384.

Herschell, A. D., Kogan, I. N., Celedonia, K. L., Gavin, I. G., \& Stein, B. D. (2009). Understanding community mental health administrators' perspectives on dialectical behaviour therapy implementation. Psychiatric Services, $60,985-988$

Herschell, A.D., Lindheim, O.I., Kogan, I.N., Celedonia, K.L., Stein, B.D. (2014). Evaluation of an implementation initiative for embedding dialectical behaviour therapy in community settings. Evaluation Program Planning, 43, 55-63

King, I., Hibbs, R. A. B., Saville, C. W. N. \& Swales, M. A. (2018). The sustainability of Dialectical Behaviour Therapy Programmes: A mixed methods analysis of barriers and facilitators to implementation within UK health care settings. BMC Psychiatry, 18:302.

Kinsey, K., \& Reed, P. G. (2015). Linking Native American tribal policy to practice in mental health care. Nurs Sci Q., doi:10.1177/0894318414558616.

Koons, C. R., Chapman, A. L., Betts, B. B., O'Rourke, B., Morse, N., \& Robins., C. I. (2006). Dialectical behaviour therapy adapted for the vocational rehabilitation of significantly disabled mentally ill adults. Cognitive and Behavioural Practice, 13(2), 146-156.

Krawitz, R. \& Miga, E. M. (2019). Financial cost-effectiveness of dialectical behaviour therapy (DBT) for borderline personality disorder (BPD). In Swales, M. (Ed) Oxford Handbook of DBT. Oxford: OUP.

Linehan, M. M. (1993). Cognitive-behavioral treatment for borderline personality disorder. New York: Guilford Press.

Linehan, M. M. (2015). DBT Skills Training Manual. Second Edition. New York: Guilford Press.

Linehan, M. M., Armstrong, H. E., Suarez, A., Allmon, D., \& Heard, H. L. (1991). Cognitive-behavioral treatment of chronically parasuicidal border line patients. Archives of General Psychiatry, 48(12), 1060-1064.

Linehan, M. M., Cochran, B.N. \& Kehrer, C.A. (2001). Dialectical behaviour therapy for borderline personality disorder. In D. H. Barlow (Ed.), Clinical handbook of psychological disorders: A step-by-step treatment manual. New York: The Guilford Press.

Linehan, M. M., Korslund, K. E., Harned, M. S., Gallop, R. I., Lungu, A., Neacsiu A. D., McDavid, I., Comtois, K. A \& Murray-Gregory, A. M. (2015). Dialectical behavior therapy for high suicide risk in individuals with borderline personality disorder: a randomized clinical trial and component analysis. JAMA Psychiatry, 72 (5), 475-82.

Little, L., Butler, L. S., \& Fowler, I. (2010). Change from the ground up: Bringing informed-dialectical behavioral therapy to residential treatment. Residential Treatment of Children and Youth, 27(2):80-91.

Mann, I. I., Apter, A., Bertolote, I., Beautrais, A., Currier, D., Haas, A., .. Hendin, H. (2005). Suicide Prevention Strategies: A systematic review. IAMA, 294, 2064-2074.

McCauley, E., Berk, M. S., Asarnow, I. R., Adrian, M., Cohen, I., Korslund, K., Avina, C., Hughes I., Harned, M., Gallop, R. \& Linehan, M. M. (2018). Efficacy of Dialectical Behaviour Therapy for Adolescents at High Risk for Suicide: A Randomized Clinical Trial. IAMA Psychiatry, 75(8), 777-785.

McHugh, R. K. \& Barlow, D.H. (2010). The dissemination and implementation of evidence-based psychological treatments: A review of current efforts. American Psychologist, 65(2), 73-84

McMain, S. F., Chapman, A. L, Kuo, J. R., Guimond, T., Streiner, D. L., DixonGordon, K. L., Isaranuwatchai, W., \& Hoch, I. S. (2019). The effectiveness of 6-month versus 12-months of dialectical behaviour therapy for borderline personality disorder: the feasibility of a shorter treatment and evaluating responses (FASTER) trial. BMC Psychiatry, 18:230.

Mehlum, L. (2019). DBT as a suicide and self-harm treatment: Assessing and treating suicidal behaviours. In Swales, M. (Ed). Oxford Handbook of DBT. Oxford: OUP.

Mehlum, L., Tormoen, A., Ramberg, M., Haga, E., Diep, L. M., Laberg, S., Larsson, B., Stanley, B. H., Miller, A. L., Sund, A. M., \& Groholt, B. (2014) Dialectical behavior therapy for adolescents with recent and repeated self-harming behavior-first randomized controlled trial. Journal of the American Academy of Child and Adolescent Psychiatry, 53, 1082-1091.
Miga, E. M., Neacsiu, A. D, Heard, H. L. \& Dimeff, L.A. (2019). Dialectical Behaviour Therapy from 1991-2015: What do we know about clinical efficacy and research quality? In Swales, M. (Ed). Oxford Handbook of DBT. Oxford: OUP.

Miller, A. L., Rathus, I. H. \& Linehan, M. M. (2007). Dialectical Behaviour Therapy for Suicidal Adolescents. New York: Guilford Press.

National Institute for Health and Clinical Excellence (2009). Borderline personality disorder: recognition and management. NICE guideline (CG78).

Navarro-Haro, M. V., Harned, M. S., Korslund, K. E., DuBose, A., Chen, T., Ivanoff. A. \& Linehan, M. M. (2019). Predictors of adoption and reach following Dialectical Behaviour Therapy Intensive Training ${ }^{\mathrm{TM}}$, Community Mental Health Journal, 55 (1), 100-111.

Oud, M., Arntz, A., Hermens, M. L. M., Verhoef, R. \& Kendall, T. (2018). Specialized psychotherapies for adults with borderline personality disorder: A systematic review and meta-analysis. Australian and New Zealand Journal of Psychiatry, 52(10), 949-961.

Perseius, K.-I., Kåver, A., Ekdahl, S., Åsberg, M., \& Samuelsson, M. (2007) Stress and burnout in psychiatric professionals when starting to use dialectical behavioural therapy in the work with young self-harming women showing borderline personality symptoms. I Psychiatry and Mental Health Nursing, 14(7), 635-643.

Pompili, M., Girardi, P., Ruberto, A., \& Tartarelli, R. (2005). Suicide in borderline personality disorder: A meta-analysis. Nordic Journal of Psychiatry, 59, 319-324.

Powell, B. I., McMillen, I. C., Proctor, E. K., Carpenter, C. R., Griffey, R. T., Bunger, A. C., Glass, I. E. \& York, I. L. (2012). A Compilation of Strategies for Implementing Clinical Innovations in Health and Mental Health. Medical Care Research Review, 69(2): 123-157.

Ramaiya, M. K., Fiorillo, D., Regmi, U., Robins, C. I., \& Kohrt, B. A. (2017). A cultural adaptation of DBT in Nepal. Cognitive Behavioural Practice, 24(4), 428-444.

Rizvi, S. L. (2019). Chain analysis in Dialectical Behaviour Therapy. New York: Guilford.

Rizvi, S. L. \& Roman, K. M. (2019). Generalization Modalities: Taking the Treatment out of the Consulting Room-Using Telephone, Text and Email. In Swales, M. (Ed). Oxford Handbook of DBT. Oxford: OUP.

Sayrs, I. \& Linehan, M. M. (2019). DBT® Teams: Development and Practice. New York: Guilford Press.

Stoffers-Winterling, I. M., Völlm, B. A., Rücker, G., Timmer, A., Huband, N., \& Lieb, K. (2012). Psychological therapies for people with borderline personality disorder. Cochrane Database of Systematic Reviews, 8(2).

Swales, M. A. (2010). Implementing Dialectical Behaviour Therapy: Organizational Pre-Treatment. The Cognitive Behaviour Therapist, 3(04), $145^{-157 .}$

Swales, M. A. \& Dunkley, C. (2019). Structuring the Wider Environment \& the DBT Team: Skills for DBT Team Leads. In Swales, M. (Ed). Oxford Handbook of DBT. Oxford: OUP.

Swales, M. A., Taylor, B., \& Hibbs, R. A. (2012). Implementing dialectical behaviour therapy: Programme survival in routine healthcare settings. Journal of Mental Health, 21(6), 548-555.

Toms, G., Williams, L., Rycroft-Malone, I., Swales, M., \& Feigenbaum, I. (2019). The development and theoretical application of an implementation framework for dialectical behaviour therapy: a critical literature review. Borderline Personality Disorder and Emotion Dysregulation, 6, 2.

Valentine, S. E., Bankoff, S. M., Poulin, R. M., Reidler, E. B. \& Pantalone, D. W. (2015). The use of dialectical behaviour therapy skills training as a standalone treatment: A systematic review of the outcome literature. Journal of Clinical Psychology, 71(1), 1-20.

Walton, C. \& Comtois, K. (2019). DBT in routine clinical settings. In Swales, M. (Ed). Oxford Handbook of DBT. Oxford: OUP.

Wang, V., Maciejewskia, M. L., Helfrich, C. D., \& Weiner, B. I. (2018). Working smarter not harder: Coupling implementation to deimplementation. Healthcare 6 (2018) 104-107.

Wolpow, S., Porter, M., \& Hermanos, E. (2000). Adapting a dialectical behavior therapy (DBT) group for use in a residential program. Psychiatric Rehabilitation Journal, 24(2), 135-141. 